\title{
Scattering Off of an Instanton
}

\author{
Richard Montgomery \\ Mathematics Department, University of California, Berkeley, CA 94720, USA
}

\begin{abstract}
We consider the scattering of a classical colored particle off an instanton. That is, we investigate Wong's equations (or equivalently, the Kaluza-Klein geodesic equations) for a color $S U(2)$ particle under the influence of a Euclidean instanton. We solve the equations in the limit in which the instanton becomes singular. Our main result is that particles with head-on trajectories scatter off the instanton with a scattering angle of $\pi / 3$. This angle is independent of the magnitude of the color charge and velocity of the particle as long as both are nonzero. The plane in which the scattering takes place is determined by the particle's initial position and color charge. We also solve for the geodesics for the corresponding (singular) Kaluza-Klein metric on $S^{7}$.
\end{abstract}

\section{Wong's Equations}

\section{Some History}

Wong (1970) introduced equations of motion for a classical colored spinless particle under the influence of an external Yang-Mills potential $A$. The equations reduce to the Lorentz equations in the abelian case. See Arodz (1982) or Balachandran et al. (1983) for further discussion.

Kaluza-Klein (1921) proposed an alternative framework in which to describe such a particle. In their framework the particle travels in a geodesic relative to a certain metric on a principal bundle over space-time. Kerner (1968) generalized Kaluza-Klein's idea from the abelian to the non-abelian case. The Wong and the Kaluza-Klein formulations were symplecticized by Sternberg (1978) and Weinstein (1978) respectively. See Sniatycki (1979) or Montgomery (1984) for a further discussion of symplectic aspects.

\section{Wong's Equations over a General Manifold}

Let $X$ be a Riemannian manifold with metric $g$. We think of $X$ as the space on which our classical colored particle travels. Let $G$ be a compact Lie group and $g$ its Lie algebra. We think of the dual $\mathrm{g}^{*}$ of the Lie algebra as the space of "color charges," or internal degrees of freedom, for our colored particle. Fix a bi-invariant 
metric $\kappa$ on $\mathfrak{g}$ and so identify $\mathfrak{g}$ with $\mathrm{g}^{*}$. Let $G \rightarrow P \rightarrow X$ be a principal bundle over $X$ with structure group $G$. Let $A$ be a connection on $P$. A is the Yang-Mills potential under whose influence our particle travels. $F$ will denote the curvature of $A$ and $D$ the covariant derivative with respect to $A$. Let $\nabla$ be the Levi-Civita connection on $X$ corresponding to $g$.

Wong's equations are equations for a curve $Q(t)$ in the adjoint bundle $\operatorname{Ad}(P)=P x_{\text {Ad }} g$ over $X$. Let $\gamma(t)$ denote the projection of $Q(t)$ onto $X$, with $\dot{\gamma}=d \gamma / d t$. We can think of $Q(t)$ as a section of $\operatorname{Ad}(P)$ along $\gamma$. Wong's equations are:

$$
\begin{gathered}
\nabla_{\dot{\gamma}} \dot{\gamma}=\langle Q, F \cdot \dot{\gamma}\rangle, \\
D_{\dot{\gamma}} Q=0 .
\end{gathered}
$$

The right-hand side of (W1) is the non-Abelian Lorentz force term. It is a vector field along $\gamma$ defined as follows. $F$ is a two-form with values in $\operatorname{Ad}(P)$, so $F \cdot \dot{\gamma}=-i_{\dot{\gamma}} F$ is a one-form along $\gamma$ with values in the same. Contracting $F \cdot \dot{\gamma}$ with $Q$ via $\kappa$ [which induces a fiber metric on $\operatorname{Ad}(P)]$ yields a standard one-form along $\gamma$. Finally, this one-form's indices can be raised by using the metric $g$, yielding the vector field $\langle Q, F \cdot \dot{\gamma}\rangle$ along $\gamma$.

Equation (W1) is the equation of a particle under the influence of a generalized Lorentz force parameterized by the color charge $Q$. Equation (W2) says that the color charge is parallel translated along this spatial trajectory. The norm $\|Q\|^{2}$ of the color charge is always a constant of the motion. This is a kinematic constraint, independent of the external potential $A$.

We will also need the first-order, coordinate form of Wong's equations

$$
\begin{gathered}
d x^{\mu} / d t=p^{\mu}, \\
d p_{\mu} / d t=-1 / 2\left(\partial g^{\alpha \nu} / \partial x^{\mu}\right) p_{\alpha} p_{v}+Q_{a} F^{a}{ }_{\mu \beta}(x) p^{\beta}, \\
d Q_{a} / d t=-Q_{d} c^{d}{ }_{a b} A^{b}{ }_{\mu}(x) p^{\mu},
\end{gathered}
$$

where $p_{\mu}=g_{\mu \nu}(x) p^{v}$. We have taken the mass and various other units equal to 1 . Spatial indices (i.e. indices referring to $X$ ) are Greek: $\mu, \beta$, etc. and Lie algebra indices are Roman: $a, b$, etc. The $x^{\mu}$ are the particle's spatial coordinates. The $p_{\mu}$ are its spatial (as opposed to canonical) momenta. The $Q_{a}$ coordinatize the particle's color-charge, i.e. they are linear coordinates on $\mathfrak{g}^{*}$ and so fiber coordinates on the co-adjoint bundle (or via $\kappa$ on the adjoint bundle). The $c^{d}{ }_{a b}$ are the structure constants for the Lie algebra relative to this basis. The $F^{a}{ }_{\mu \beta}$ are the components of the Yang-Mills field strength. Spatial indices are lowered by $g$.

This coordinate version of Wong's equations defines an o.d.e. on the vector bundle $T^{*} X \oplus \operatorname{Ad} P$, the Whitney sum of two vector bundles over $X . T^{*} X \oplus \operatorname{Ad} P$ is the phase space for Wong's equations. It is coordinatized by $\left(x^{\mu}, p_{v}, Q^{a}\right)$. The $x^{\mu}$ are coordinates for $X$, the $p_{v}$ are fiber coordinates on $T^{*} X$, and the $Q_{a}$ are fiber coordinates for $\operatorname{Ad}(P)$.

Remarks. 1. If the $Q_{a}$ are all zero, then Wong's equations reduce to the geodesic equations on $X$.

2. If $G$ is $U(1)$ then the equations reduce to the Lorentz equations.

3. The Wong phase space $T^{*} X \oplus \operatorname{Ad} P$ is equal to the pullback of the adjoint bundle $\operatorname{Ad}(P) \rightarrow X$ to $T^{*} X$ by the cotangent projection $T^{*} X \rightarrow X$. In Montgomery (1984) the Wong phase space is always referred to as this pullback bundle. 


\section{Kaluza-Klein Formulation}

There is an alternative formulation of the dynamics of our "classical quark" due to Kaluza-Klein and generalized to the non-abelian case by Kerner (1968). The equations of motion are the geodesic equations on the principal bundle $P$ with respect to a metric $d s_{A}{ }^{2}$. Intrinsically $d s_{A}{ }^{2}$ is defined by declaring that (1) the vertical and horizontal (with respect to $A$ ) subspaces are orthogonal, (2) that the fiber inclusion $G \hookrightarrow P$ is a Riemannian embedding, and (3) that the projection $\pi: P$ $\rightarrow X$ is a Riemannian submersion. Locally

$$
d s_{A}{ }^{2}=g_{\mu v}(x) d x^{\mu} d x^{v}+\kappa_{a b}\left(\theta^{a}+A^{a}{ }_{\mu}(x) d x^{\mu}\right)\left(\theta^{b}+A^{b}{ }_{v}(x) d x^{\nu}\right),
$$

where the $\theta^{a}$ are the basis of right invariant one-forms on $G$ dual to our coordinate basis for $g$. Note that the structure group $G$ acts by isometries relative to this metric.

The two formulations are related as follows. Let $\sigma(t)$ be a curve in $P$. Consider $A_{\sigma(t)} \dot{\sigma}(t) \in \mathfrak{g}$, the vertical projection of the velocity $\dot{\sigma}$. Set $Q(t)=\left[\sigma(t), A_{\sigma(t)} \dot{\sigma}(t)\right] \in \mathrm{Ad}(P)$. Then $\sigma(t)$ is a geodesic w.r.t. $d s_{A}{ }^{2}$ iff $Q(t)$ is a solution to Wong's equations with the potential $A$.

Symmetries. This paper relies heavily on symmetry groups. By a symmetry we will mean a bundle automorphism of $P$ which

(i) covers an isometry on the base space $X$,

(ii) preserves the connection $A$.

Clearly such an automorphism is an isometry for $d s_{A}{ }^{2}$.

Any automorphism of $P$ induces an automorphism of the Wong phase space $T^{*} X \oplus \mathrm{Ad} P$. An automorphism of the Wong phase space which is induced by a symmetry will be called a symmetry of Wong's equations. It is clear from the above correspondence between solutions of the Kaluza-Klein and Wong's equations that a symmetry of Wong's equations maps solutions of the equations to solutions to the equations.

\section{Hamiltonian Formalism}

Some Hamiltonian formalism will be used in arriving at our scattering result. Here we summarize the formalism for Wong's equations. The Poisson brackets for Wong's equations are non-canonical. The Hamiltonian for Wong's equations is the particle's kinetic energy

$$
H=1 / 2 g^{\mu v}(x) p_{\mu} p_{v} .
$$

Define the canonical momenta by the minimal coupling procedure:

$$
p_{\mu}^{\mathrm{can}}=p_{\mu}+Q_{a} A_{\mu}^{a}(x) .
$$

Then in the $\left(x^{\mu}, p^{\mathrm{can}}, Q_{a}\right)$ coordinates the Poisson brackets are given by

$$
\left\{x^{\mu}, p^{\text {can }}{ }_{v}\right\}=\delta^{\mu}{ }_{\nu},\left\{Q_{a}, Q_{b}\right\}=c^{d}{ }_{\text {ad }} Q_{d}, \quad \text { all other brackets zero. }
$$

These relations can be used to find formulas for the brackets of the $\left(x^{\mu}, p_{v}, Q_{a}\right)$. Hamilton's equations are given by $d f / d t=\{f, H\}$ for $f$ any function on $T^{*} X \oplus \operatorname{Ad}(P)$. In particular if we take for $f$ the coordinate functions $x^{\mu}, p_{v}$, and $Q_{a}$ then Hamilton's equations are (W1a), (W1b), and (W2a) respectively. See Montgomery (1984) for more details. 
There is a kinematic constraint associated with the brackets of the $Q$, namely the $Q$ must remain on whatever adjoint orbit they started on, independent of the choice of Hamiltonian $H$. For the regular orbits this constraint is seen by the fact that any adjoint-invariant smooth function of the $Q s$ is a Casimir: its Poisson bracket with all other functions is zero. One such Casimir is $\|Q\|^{2}=\kappa^{a b} Q_{a} Q_{b}$. For $G=S U(2)$ this is the only Casimir.

Remark. The $\left(x^{\mu}, p^{\text {can }}{ }_{v}, Q_{a}\right)$ are best thought of as coordinates on the Poisson reduced manifold $T^{*} P / G$. The symplectic leaves of this Poisson manifold are the "universal phase spaces" of Weinstein (1978). As mentioned the $\left(x^{\mu}, p_{v}, Q_{a}\right)$ are coordinates on $T^{*} X \oplus \operatorname{Ad}(P)$. The symplectic leaves of this Poisson manifold are Sternberg's (1977) phase spaces. The minimal coupling procedure (1.2) is an isomorphism between these two Poisson manifolds. For more details see Montgomery (1984).

\section{Momentum Maps}

Momentum maps are the Hamiltonian version of Noether conserved quantities. Suppose we have a left action of a Lie group $K$ on $P$ by symmetries, where symmetries are as defined above. This action induces an action of $K$ on the Wong phase space $T^{*} X \oplus \mathrm{Ad} P$ which leaves the Hamiltonian invariant, and is canonical (bracket preserving).

The action on $T^{*} X \oplus \operatorname{Ad} P$ has a momentum map $J: T^{*} X \oplus \operatorname{Ad} P \rightarrow \mathfrak{f}^{*}$. Here $\mathfrak{f}$ denotes the Lie algebra of Kand $\mathfrak{f}^{*}$ is its dual. For $\xi \in \mathfrak{l}$, let $\xi_{E}$ denote the corresponding infinitesimal generator, a vector field on $E$. Let $J^{\xi}(e)=\langle J(e), \xi\rangle$. Then $J$ is essentially defined by the relation

$$
d f \cdot \xi_{E}=\left\{f, J^{\xi}\right\} \text { for all smooth function } f \text { on } E .
$$

$J$ is a conserved vector for the dynamics, since $\left\{J^{\xi}, H\right\}=-d H \cdot \xi_{E}=$ $-d\left\{(\exp \lambda \xi)^{*} H\right\} / d \lambda=0$.

The formula for $J$ in our local coordinates is

$$
J^{\xi}(x, p, Q)=\left\langle p^{\text {can }}, \xi_{X}(x)\right\rangle+\left\langle Q, \xi_{P}(x)\right\rangle .
$$

Here $\xi_{X}(x) \in T_{x} X$ is the infinitesimal generator for the action on $X . \xi_{P}(x) \in \operatorname{Ad}_{x} P$ is the infinitesimal generator of the $K$ action on $P$. It represents an infinitesimal automorphism of $P$. In a local trivialization $\xi_{P}(x) \in \mathfrak{g}$ and the infinitesimal automorphism has the form $g \mapsto \xi_{P}(x) g, g \in G$.

\section{Our Problem and the Statement of its Solution}

For the rest of this paper we take $G \rightarrow P \rightarrow X$ to be the quaternionic Hopf fibration $S p(1) \rightarrow S^{7} \rightarrow S^{4}$. Recall that $S p(1)$ is isomorphic to $S U(2)$ which is topologically $S^{3}$. We take the standard metrics on the fiber $S^{3}$ and on the base $S^{4}$, with radii 2 and 1 respectively, and we take $A$ to be a self-dual connection for this bundle, i.e. an instanton with instanton number 1 . We will begin by reviewing instantons. For further details regarding instantons we suggest Freed and Uhlenbeck (1984, pp. 100-105). 


\section{Review of Instantons}

Let $\mathbf{H}=\mathbf{R}^{4}$ denote the space of quaternions. Throughout this paper we identify $S^{4}$ with $\mathbf{H} \cup\{\infty\}=$ quaternionic projective space $\mathbf{H P}^{1}$, via stereographic projection so that $\infty$ corresponds to the north pole and 0 to the south pole. $\mathbf{H P}^{1}$ is the space whose points consist of right quaternionic lines in $\mathbf{H} \times \mathbf{H}$. We write points of $\mathbf{H P}^{1}$ in homogeneous coordinates: $\left[q_{1}, q_{2}\right]=\left[q_{1} h, q_{2} h\right]$. (Caveat: Freed and Uhlenbeck use left quaternionic lines, hence many of their formulas are the quaternionic conjugate of ours.) The affine coordinate of a point $[x, 1] \in \mathbf{H P}^{\mathbf{1}}$ is $x \in \mathbf{H}$. The point $[1,0]$ corresponds to $\infty$. In this manner $\mathbf{H P}^{1}=\mathbf{H} \cup\{\infty\}$. The standard metric on $S^{4}$ is then

$$
g(x)=\left(\frac{2}{1+\|x\|^{2}}\right)^{2} \operatorname{Re}\{d x d \bar{x}\}, \quad x \in \mathbf{H} .
$$

Consider $S^{7} C \mathbf{H} \times \mathbf{H}$ as consisting of pairs $\left(q_{1}, q_{2}\right)$ with

$$
\left\|q_{1}\right\|^{2}+\left\|q_{2}\right\|^{2}=r^{2} \text {. }
$$

[The correct choice for $r$ is 2. This insures that the Kaluza-Klein metric corresponding to the central instanton (see below) is the standard metric on $S^{7}(2)$.] The action of the structure group is given by $\left(q_{1}, q_{2}\right) \cdot g=\left(q_{1} g, q_{2} g\right)$.

The Hopf projection $S^{7} \rightarrow S^{4}$ is simply given by $\left(q_{1}, q_{2}\right) \mapsto\left[q_{1}, q_{2}\right]$. The standard local section $s$ over $\mathbf{H}$ is

$$
s(x)=\frac{r}{\sqrt{1+\|x\|^{2}}}(x, 1) .
$$

The corresponding local trivialization over $\mathbf{H}$ is $(x, g) \mapsto s(x) \cdot g$, which is a diffeomorphism, $\mathbf{H} \times S p(1) \rightarrow S_{\mathbf{H}}^{7}$.

Every self-dual connection for the quaternionic Hopf fibration is characterized up to gauge equivalence by its center $b \in S^{4}$ and its width $\lambda, 0<\lambda \leqq 1$. This is a fundamental result due to Atiyah et al. (1978). The space of gauge equivalence classes of self-dual connections is known as the moduli space. Topologically the moduli space is a unit 5-ball, with $(b, \lambda) \in S^{4} \times(0,1)$ corresponding to $(1-\lambda) b \in B^{5}$. The connections with width 1 are thus all gauge equivalent and correspond to the center of the ball. The Kaluza-Klein metric corresponding to this central connection is the standard metric on $S^{7}$.

A point $(b, 0)$ on the boundary represents a singular connection with singularity at $b \in S^{4}=\partial B^{5}$. Our goal in this paper is to solve the Wong system corresponding to such a singular connection. The $S O(5)$ symmetry of the situation allows us to reduce the problem to the case $b=0=$ south pole. See Remark 3 at the end of this section. From now on we will assume $b=0$.

After being pulled back by our standard local sections the connection $A_{\lambda}$ with width $\lambda$ and center $b=0$ is given by

$$
s^{*} A_{\lambda}(x)=\frac{1}{\lambda^{2}+\|x\|^{2}} \operatorname{Im}\{\bar{x} d x\}, \quad x \in \mathbf{H} .
$$

We have identified the Lie algebra of $S p(1)$ with the pure imaginary quaternions Im $\mathbf{H}$. In the local trivialization over $\mathbf{H}$

$$
A_{\lambda}(x, g)=\bar{g} \frac{\{\operatorname{Im}\{\bar{x} d x\}+d\} g}{\lambda^{2}+\|x\|^{2}},
$$


The curvature of $A_{\lambda}$ is given by

$$
s^{*} F_{\lambda}(x)=\frac{\lambda^{2}}{\left(\lambda^{2}+\|x\|^{2}\right)^{2}} \operatorname{Im}\{d \bar{x} \wedge d x\} .
$$

For each $\lambda$ we then have a system $W_{\lambda}$ of Wong's equations over $\mathbf{H} \cup\{\infty\}$. Our problem is then to solve these Wong's equations in the limit $\lambda \rightarrow 0$. If we simply set $\lambda=0$ then both the connection and its curvature are singular at $x=0$. The curvature in fact acts like a $\delta$-function. The Wong system $W_{0}$ is thus ill-defined at $x=0$.

Naively, our classical quark acts like a charged particle travelling in a magnetic field $B(x)=\delta(x) B_{0}$. As long as the particle misses the origin there is no force on it so it travels in a geodesic. However if it goes through the origin it is instantaneously subject to an infinite deflecting force. In general it would seem that the particle's trajectory would no longer be well-defined after it passes through the origin. However if the $\delta$-function is "nice enough" the particle should scatter by some finite angle and continue along on a geodesic. This is the case for our classical quark: the scattering angle in $\pi / 3$ and the scattering plane is determined by the quark's initial position and internal degree of freedom.

Recall from the previous section that Wong's equations can be written as o.d.e. $S$ on the vector bundle $T^{*} S^{4} \oplus \mathrm{Ad} S^{7}$ over $S^{4}$. Our local trivialization of $S_{\mathbf{H}}^{7}$ over $\mathbf{H}$ $C S^{4}$ induces a local trivialization $\operatorname{Ad}\left(S^{7}\right)_{\mathbf{H}} \simeq \mathbf{H} \times \operatorname{Im} \mathbf{H}$. The Wong phase space in this local trivialization is then $\left(T^{*} S^{4} \oplus A d S^{7}\right)_{\mathbf{H}} \simeq \mathbf{H} \times \mathbf{H} \times \operatorname{Im} \mathbf{H}$, and a typical element in it will be written $(x, p, Q)$. Set

$$
\beta=\|x\|^{2} \text {. }
$$

Then Wong's equations [W1a, b, W2] for the instanton $A_{\lambda}$ read:

$$
\begin{gathered}
\dot{x}=1 / 4(1+\beta)^{2} p, \\
\dot{p}=-1 / 2(1+\beta)\|p\|^{2} x-\frac{\lambda^{2}}{2} \frac{(1+\beta)^{2}}{\left(\lambda^{2}+\beta\right)^{2}} p Q \\
\dot{Q}=-1 / 4 \frac{(1+\beta)^{2}}{\lambda^{2}+\beta}[\bar{x} p, Q] .
\end{gathered}
$$

Here means $d / d t$, and the brackets $[\cdot, \cdot]$ are the Lie brackets in $\mathbf{H}$ : $[q, h]=q h-h q$, which is always an element of $\operatorname{Im} \mathbf{H}$.

Initial conditions at time $t=t_{0}$ for our Wong's equations are given by $\left(x_{0}, p_{0}, Q_{0}\right) \in \mathbf{H} \times \mathbf{H} \times \operatorname{Im} \mathbf{H}$. We will always assume that $x_{0} \neq 0$. Let $(x(t, \lambda), p(t, \lambda)$, $Q(t, \lambda))$ be the solution to the Wong system $W_{\lambda}$ with these initial conditions. $B y$ definition the solution to Wong's equations for the singular connection $A_{0}$ and the same initial conditions $\left(x_{0}, p_{0}, Q_{0}\right)$ is the path $(x(t), Q(t))=\lim _{\lambda \rightarrow 0}(x(t, \lambda), Q(t, \lambda))$ in $\mathbf{H} \times \operatorname{Im} \mathbf{H}$. We allow $x(t)=\infty$ corresponding to the north pole. We also write $p(t)=\lim _{\lambda \rightarrow 0} p(t, \lambda)$ however as we will see this limit does not exist when $x(t)=0$ (see Fig. 1).

Away from $x=0$,

$$
F_{\lambda} \rightarrow 0 \quad \text { and } \quad A_{\lambda} \rightarrow A_{0}=\operatorname{Im}\left\{\bar{x} d x /\|x\|^{2}\right\} .
$$


Wong's equations (W1,2) of Sect. 1 then become

$$
\nabla_{\dot{\gamma}} \dot{\gamma}=0 \quad \text { and } D^{0} \dot{\gamma}=0 \text { for } \gamma(t) \neq 0 \text {, }
$$

where $D^{0}$ is the covariant derivative for $A_{0}$. Let $(x(t, 0), p(t, 0), Q(t, 0))$ denote the solution to these equations with initial conditions $\left(x_{0}, p_{0}, Q_{0}\right)$. The convergence (2.5) is uniform over compact sets of $\mathbf{H} \cup \infty \backslash\{0\}=S^{4} \backslash\{0\}$. A standard argument using Gronwall's lemma then says that $(x(t), p(t), Q(t))=(x(t, 0), p(t, 0), Q(t, 0))$ as long as $x(t, 0) \neq 0$.

The curves $x(t, 0)$ are just the stereographic projections of arcs of great circles. As long as $x_{0} \neq \infty$ and $p_{0} \neq c x_{0}$ with $c$ real, these circles do not go through the south pole, $x=0$, and so $x(t)$ corresponds (under stereographic projection) to a great circle for all t. $Q(t)$ is parallel translated around this circle w.r.t. the connection $A_{0}$. Since the curvature, $F_{0}=0$ away from 0 it follows that $Q\left(t_{0}+T\right)=Q_{0}$ where $T$ is the geodesic's period

$$
T=\frac{2 \pi}{\left\|\dot{x}\left(t_{0}\right)\right\| S^{4}}=\frac{4 \pi}{1+\beta_{0}} \frac{1}{\left\|p_{0}\right\|} .
$$

We will call the exceptional initial conditions

$$
\text { scattering data }=\left\{\left(x_{0}, p_{0}, Q_{0}\right): x_{0}=\infty \text { or } p_{0}=c x_{0} \text { with } c \text { real }\right\} .
$$

The corresponding curves, $x(t)$ are the interesting ones, since they go through the singularity.

Proposition 1. Assume that $x_{0}, p_{0}$, and $Q_{0}$ are all nonzero. Then the solution $(x(t), Q(t))$ as defined above to Wong's equations for the singular connection with initial conditions $\left(x_{0}, p_{0}, Q_{0}\right)$ exists for all $t$ and is given as follows:

If $\left(x_{0}, p_{0}, Q_{0}\right)$ are scattering data then the trajectory is as in Fig. $1: x(t)$ consists of 6 rays through the origin. These rays all lie in the 2-plane $P$ spanned by $x_{0}$ and $x_{0} Q_{0}$. The rays are travelled by $x(t)$ in such a way that the angle between a successive incoming and outgoing ray is $\pi / 3$ relative to the orientation $\left\{x_{0}, x_{0} Q_{0}\right\}$ of the 2-plane. At $\infty$ (the north pole) the great circles are traversed in the standard way: there is no discontinuity in $x(t)$ 's derivative at $\infty$. On $S^{4}$ this trajectory is the union of three great circles through the south pole. The trajectory is periodic with period $3 T$, with $T$ given by (2.7). $Q(t)=Q_{0}$ is constant throughout.

If $\left(x_{0}, p_{0}, Q_{0}\right)$ are not scattering data then $x(t)$ is the stereographic projection of a great circle on $S^{4} . Q(t)$ is parallel translated along this geodesic w.r.t. the connection $A_{0}$. All such trajectories are periodic with period $T$.

Outline of Proof. The proof of the theorem for non-scattering data follows from Gronwall's estimate, as mentioned above. Proving the theorem for scattering data occupies the rest of this paper. A priori, it is not even clear that $\lim _{\lambda \rightarrow 0} x(t, \lambda)$ exists for $t>t_{c}$ where $x\left(t_{c}, 0\right)=0$. This proof is broken into two parts. In the first part, presented in the next section, we reduce the problem to an o.d.e. in the plane. This is done by using the fact that $S p(1) \times S p(1)$ acts as a symmetry group for the Wong's equations $\left(W_{\lambda}\right), \lambda>0$. In the second part, presented in Sect. 4, we analyze this o.d.e. in the limit $\lambda \rightarrow 0$ by using methods from classical scattering theory and singular perturbation theory. 


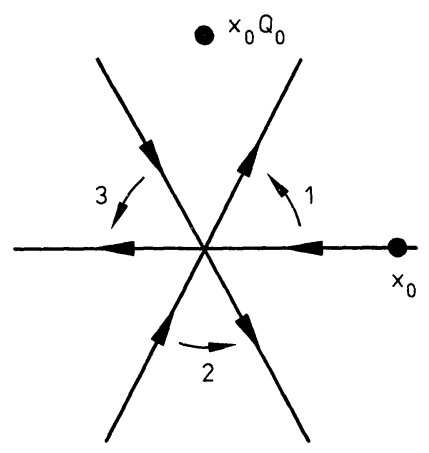

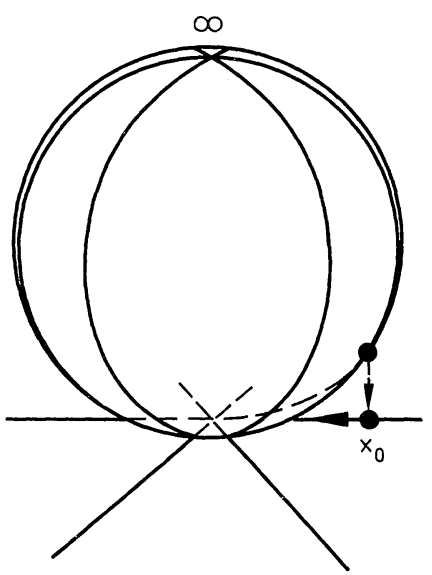

Fig. 1

Remarks. 1. We will discuss what the scattering looks like up on the bundle in Sect. 5. That is, what are the geodesics for the singular Kaluza-Klein metric on $S^{7}$ ? When the curve passes through the fiber $S^{3} C S^{7}$ over the singularity 0 it jumps along an arc of a great circle of the fiber. The angle subtended by this arc is $\pi / 3$.

2. The fact that $Q(t)$ is constant on the scattering trajectories is globally consistent even though $\operatorname{Ad}\left(S^{7}\right)$ is a nontrivial bundle over $S^{4}$. From a topological point of view this constancy of $Q(t)$ is possible because the vector bundle $\operatorname{Ad}\left(S^{7}\right)$ $\rightarrow S^{4}$ is trivial over every $S^{2}$ in $S^{4}$.

3. By symmetry, the proposition also holds for all the other singular self-dual instantons with instanton number 1 connections over $S^{4}$. Such a connection is represented by a point $(b, 0)$. We define the Wong dynamics just as in the case $b=0$ : it is the limit $\lambda \rightarrow 0$ of the one-parameter family of Wong dynamics corresponding to the connections $(b, \lambda)$. Let $R \in S O(5)$ be any isometry of $S^{4}$ which takes 0 (the south pole) to $b . R$ is covered by an automorphism $\tilde{R}$ of $S^{7}$ which takes the connections $(0, \lambda)$ to the connections $(b, \lambda)$. So the two families of Wong dynamics, and hence their limits, are conjugate by $\tilde{R}$. Thus the proposition above holds for any singular connection except that 0 is replaced by the singularity $b$ and $\infty$ by the point antipodal to $b$.

4. The adjoint bundle $\mathrm{Ad} S^{7}$, which is more or less the configuration space for Wong's equations is a vector bundle over $S^{4}$. Its unit sphere bundle $E$ can be identified with the twistor space [see Atiyah (1979), or Atiyah et al. (1978)] whose fiber $E_{x}, x \in S^{4}$, consists of the set of complex structures on $T_{x} S^{4} . E$ is diffeomorphic to $\mathbf{C P}^{3}$. $T^{*} S^{4} \oplus E$ can be identified with the symplectic quotient of $T^{*} S^{7}$ by the action of the structure group $S p(1)$.

5 . We chose a particular path, namely $(b, \lambda)$, approaching $(b, 0)$ in order to define the singular dynamics. If we chose a different path $(b(\lambda), \lambda), d b / d \lambda \neq 0$ for $\lambda=0$, it is likely that the singular dynamics would be different.

6. The $\pi / 3$ scattering result in Proposition 1 probably also holds for any Riemannian 4-manifold $X$ which admits concentrated instantons. A result of Taubes states that if $X$ is a simply connected spin manifold then it admits such connections. The Donaldson Theorem then says that such an $X$ is either $S^{4}$ or the connected sum of $\mathbf{C P}^{2}$ s. 


\section{Symmetries, Conserved Quantities and Reduction to an O.d.e. in the Plane}

In this section we work entirely in stereographic coordinates. The two main results of this section are:

Lemma 1. If $\left(x_{0}, p_{0}, Q_{0}\right)$ are scattering data then $x(t, \lambda)$ (and hence $p(t, \lambda)$ ) is restricted to lie in the two-plane

$$
\mathbf{P}=\operatorname{Span}_{\mathbf{R}}\left\{x_{0}, x_{0} Q_{0}\right\} \subset \mathbf{H} .
$$

(Note that since $\bar{Q}_{0}=-Q_{0}$ that $x_{0}$ and $x_{0} Q_{0}$ are orthogonal.)

Lemma 2. Let $(\varrho, \theta)$ be polar coordinates on the two-plane $P$ where the plane is oriented according to $\left\{x_{0}, x_{0} Q_{0}\right\}$. Set $\varrho^{2}=\beta$ so that

$$
\|x\|^{2}=\beta
$$

as in Eq. $(W \lambda)$ above. Then $x(t, \lambda)$ obeys the o.d.e.:

$$
\begin{gathered}
1 / 4 \dot{\beta}=2 H \beta((1+\beta) / 2)^{2}-((1+\beta) / 2)^{4} a(\beta, \lambda)^{2}, \\
\beta^{2} \dot{\theta}^{2}=((1+\beta) / 2)^{4} a(\beta, \lambda)^{2},
\end{gathered}
$$

where

$$
a(\beta, \lambda)=\frac{\lambda^{2}\left\|Q_{0}\right\|\left(\beta_{0}-\beta\right)}{\left(\lambda^{2}+\beta\right)\left(\lambda^{2}+\beta_{0}\right)} .
$$

As a corollary we will show:

Corollary 1. If $\left(x_{0}, p_{0}, Q_{0}\right)$ is scattering data then the component $Q(t, \lambda)$ of the Wong trajectory is constant: $Q(t, \lambda)=Q_{0}$ for all $t, \lambda>0$.

In proving these three results, we will omit the details of a number of calculations. These details are available upon request.

These two lemmas are obtained by using the fact that $S p(1) \times S p(1)$ acts as a symmetry group (in the sense of the first section) on the Wong system $W_{\lambda}$. An element $\left(g_{1}, g_{2}\right)$ of $S p(1) \times S p(1)$ acts on $S^{7} \subset \mathbf{H} \times \mathbf{H}$ by $\left(g_{1}, g_{2}\right) \cdot\left(q_{1}, q_{2}\right)$ $=\left(g_{1} q_{1}, g_{1} q_{2}\right)$. In the standard trivialization of $S^{7}$ over $\mathbf{H}$ this action is:

$$
\left(g_{1}, g_{2}\right) \cdot(x, g)=\left(g_{1} x g_{2}^{-1}, g_{2} g\right) \text {. }
$$

The transformation of the base, $x \mapsto g_{1} x g_{2}^{-1}$ is an element of $S O(4)$ hence an isometry of $S^{4}$. This action also preserves the connections $A_{\lambda}$ :

$$
L_{g_{1}, g_{2}} * A_{\lambda}=A_{\lambda},
$$

where $L_{g_{1}, g_{2}}$ is the transformation (3.3). Hence this action is an action by symmetries as defined in Sect. 1.

The induced action of $S p(1) \times S p(1)$ on the Wong phase space $T^{*} S^{4} \oplus \operatorname{Ad}\left(S^{7}\right)$ is given in our local trivialization by:

$$
\left(g_{1}, g_{2}\right) \cdot(x, p, Q)=\left(g_{1} x g_{2}^{-1}, g_{1} p g_{2}^{-1}, g_{2} Q g_{2}^{-1}\right) .
$$

There is a momentum map, or conserved vector, $J$ corresponding to this action (see end of Sect. 1). $J$ is a function on Wong phase space with values in the dual of the 
Lie algebra of the symmetry group. Identify this dual Lie algebra with $\operatorname{Im} \mathbf{H}$ $\times \operatorname{Im} \mathbf{H}$. Then using formula (1.3) we find that $J=\left(J_{1}, J_{2}\right)$, where

$$
\begin{gathered}
J_{1}(x, p, Q)=\operatorname{Im}(p \bar{x})+x Q \bar{x} /\left(\lambda^{2}+\|x\|^{2}\right), \\
J_{2}(x, p, Q)=-\operatorname{Im}(\bar{x} p)+\lambda^{2} Q /\left(\lambda^{2}+\|x\|^{2}\right) .
\end{gathered}
$$

From general theory we know that $J$ is conserved by the Wong dynamics $\left[W_{\lambda}\right]$, i.e. $d J / d t=0$. This can also be checked by a direct but tedious calculation.

Proof of Lemma 1. We can consider $J$ as taking values in the Lie algebra of $S O(4)$ since this Lie algebra is isomorphic to the Lie algebra of $S p(1) \times S p(1)$. Then $\left(J_{1}, J_{2}\right)$ is thought of as the skew-symmetric endomorphism

$$
h \mapsto J_{1} h-h J_{2}
$$

of $\mathbf{H}=\mathbf{R}^{4}$. Every skew symmetric endomorphism of $\mathbf{R}^{4}$ has two orthogonal invariant two-dimensional subspaces $\mathbf{P}_{+}$and $\mathbf{P}_{-}$. Consequently, these subspaces are invariants of the Wong dynamics.

Let $x_{+}$and $x_{-}$denote the projections of $x$ [the $x$ of $\left.J=J(x, p, Q)\right]$ onto these subspaces. A calculation shows that

$$
\left\|x_{+}\right\|^{2}=\alpha_{+}\|x\|^{2} \quad \text { with } \quad \alpha_{+}=\frac{1}{2}(1-\cos \mu)
$$

and

$$
\left\|x_{-}\right\|^{2}=\alpha_{-}\|x\|^{2} \quad \text { with } \quad \alpha_{-}=\frac{1}{2}(1+\cos \mu),
$$

where $\mu$ is the angle between $J_{1} x$ and $x J_{2}$, which satisfies

$$
2 \cos \mu=\frac{\|Q\|^{2}-\left(\left\|J_{1}\right\|^{2}+\left\|J_{2}\right\|^{2}\right)}{\left\|J_{1}\right\|\left\|J_{2}\right\|} .
$$

Since the $\alpha_{ \pm}$depend only on $J$ and $\|Q\|^{2}$, Eq. (3.8) are dynamical constraints: they constrain the position variable $x$ to lie on a certain cone in $\mathbf{H}$ which is generally 3-dimensional. However, on scattering data $\bar{x}_{0} p_{0}$ is real, so that $\operatorname{Im}\left\{p_{0} \bar{x}_{0}\right\}$ $=\operatorname{Im}\left\{\bar{x}_{0} p_{0}\right\}=0$ and

$$
\begin{gathered}
J_{1}\left(x_{0}, p_{0}, Q_{0}\right)=x_{0} Q \bar{x}_{0} /\left(\lambda^{2}+\left\|x_{0}\right\|^{2}\right), \\
J_{2}\left(x_{0}, p_{0}, Q_{0}\right)=\lambda^{2} Q_{0} /\left(\lambda^{2}+\left\|x_{0}\right\|^{2}\right) .
\end{gathered}
$$

It follows that

$$
\left\|J_{1}\right\|+\left\|J_{2}\right\|=\|Q\|
$$

on scattering data. Plugging this result into (3.8b) we see that $\cos \mu=1$ and hence $\alpha_{+}=0$ and $\alpha_{-}=1$. Thus $x_{0}=x_{0_{-}}$, i.e. $x_{0}$ lies on the invariant two-plane $\mathbf{P}=\mathbf{P}$. Since $\alpha_{+}$and $\mathbf{P}_{-}$are invariants of the $\lambda$-dynamics, it follows from Eq. (3.8a) that $x(t, \lambda)$ remains on $\mathbf{P}$ for all $t$.

A priori, $\mathbf{P}_{-}$depends on $\lambda$ through $J$. However, on scattering data $\mathbf{P}_{-}$is independent of $\lambda$. This is most easily seen by substituting $h=x_{0}$ and then $h=x_{0} Q_{0}$ into the endomorphism (3.7) with $J_{1}$ and $J_{2}$ given by (3.9). The result is that under this endomorphism $\operatorname{span}_{\mathbf{R}}\left\{x_{0}\right\} \mapsto \operatorname{span}_{\mathbf{R}}\left\{x_{0} Q_{0}\right\}$ and $\operatorname{span}_{\mathbf{R}}\left\{x_{0} Q_{0}\right\} \mapsto \operatorname{span}_{\mathbf{R}}\left\{x_{0}\right\}$ and thus $\mathbf{P}_{+}=\operatorname{span}_{\mathbf{R}}\left\{x_{0}, x_{0} Q_{0}\right\}$. This completes the proof of Lemma 1 . 
Proof of Lemma 2. The energy (1.1) of our system is

$$
H=\frac{1}{2}((1+\beta) / 2)^{2}\|p\|^{2}
$$

and is conserved. A calculation shows that in general

where

$$
\|p\|^{2}=\beta^{-1}\left\{\frac{1}{4}[2 /(1+\beta)]^{4} \dot{\beta}^{2}+\Phi(\beta, \lambda)\right\}
$$

$$
\Phi(\beta, \lambda)=\lambda^{2}\left\|J_{1}\right\|^{2} /\left(\lambda^{2}+\beta\right)+\beta\left\|J_{2}\right\|^{2} /\left(\lambda^{2}+\beta\right)-\lambda^{2}\|Q\|^{2} \beta /\left(\lambda^{2}+\beta\right)^{2} .
$$

Combining these results gives the equation:

$$
\frac{1}{4} \dot{\beta}^{2}=2 H \beta[(1+\beta) / 2]^{2}-[(1+\beta) / 2]^{4} \Phi(\beta, \lambda) .
$$

Using (3.9) and (3.10) we calculate that $\Phi(\beta, \lambda)=a(\beta, \lambda)^{2}$. Plugging this into (3.13) yields the first desired equation, $(3.2 \beta)$.

Remark. Equation (3.13) is the reduced dynamical equation in the sense of symplectic reduction [Arnold (1978, Appendix); Abraham and Marsden (1978, Chap. 4)]. Form the symplectic quotient of $T^{*} S^{7}$ by the group $H=S p(1) \times S p(1) \times S p(1)$. The first $S p(1)$ factor is the structure group and it acts on the right, the other two factors act on the left as in the paragraph above. A dimension count shows that the symplectic reduced space is two-dimensional. The reduced Hamiltonian thus provides a complete integral of the motion. Alternatively, form the (singular) quotient $S^{7} / H$. This is a one-dimensional Riemannian manifold coordinatized by $\beta$ and (3.13) is a form of the geodesic equation.

Since $x$ is constrained to $\mathbf{P}=\mathbf{P}_{-}$we have

$$
\|\dot{x}\|^{2}=\dot{\varrho}^{2}+\varrho^{2} \dot{\theta}^{2}
$$

in the polar coordinates $(\varrho, \theta)$. Substituting $\beta=\varrho^{2}$ yields

Now the energy

$$
\|\dot{x}\|^{2}=\frac{\dot{\beta}^{2}}{4 \beta}+\beta \dot{\theta}^{2} .
$$

$$
H=\frac{1}{2}[2 /(1+\beta)]^{2}\|\dot{x}\|^{2}
$$

is conserved. These last two equations yield

$$
\beta^{2} \dot{\theta}^{2}=2 H[(1+\beta) / 2]^{2}-\frac{1}{4} \dot{\beta}^{2} .
$$

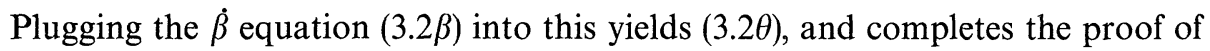
Lemma 2.

Proof of Corollary 1. Recall that Eq. $\left(W_{\lambda} 2\right)$ for $Q$ has the form $\dot{Q}=g(\beta)[\bar{x} p, Q]$. The Eqs. $(3.2 \beta, \theta)$ of Lemma 2 determine $(x(t, \lambda), p(t, \lambda))$ and are independent of $Q$ (recall that the magnitude $\|Q\|$ is automatically constant) so we can solve for $x(t, \lambda), p(t, \lambda)$ without solving for $Q(t, \lambda)$. Given these solutions, Eq. $\left(W_{\lambda} 2\right)$ becomes a linear non-autonomous o.d.e. for $Q$. Recalling the formula $\left(3.6_{2}\right)$ for the conserved vector $J_{2}$ we can rewrite the $\dot{Q}$ equation in the form $\dot{Q}=f(\beta)\left[J_{2}, Q\right]$. Initially $\operatorname{Im}(\bar{x} p)=0$ so that $\left[J_{2}, Q\right]=0 . J_{2}$ is constant of the motion. If $Q(t, \lambda)$ is also constant, then the bracket $\left[J_{2}, Q\right]$ is constant and hence zero. Thus $Q(t, \lambda)=Q_{0}$ is a solution to $\left(W_{\lambda} 2\right)$. Uniqueness of solutions implies it is the solution. 


\section{Asymptotic Analysis of the O.D.E.}

If Proposition 1 were to hold, then for $\lambda$ small, we would expect the following qualitative picture for the dynamics in the $\mathbf{P}_{+}$plane:

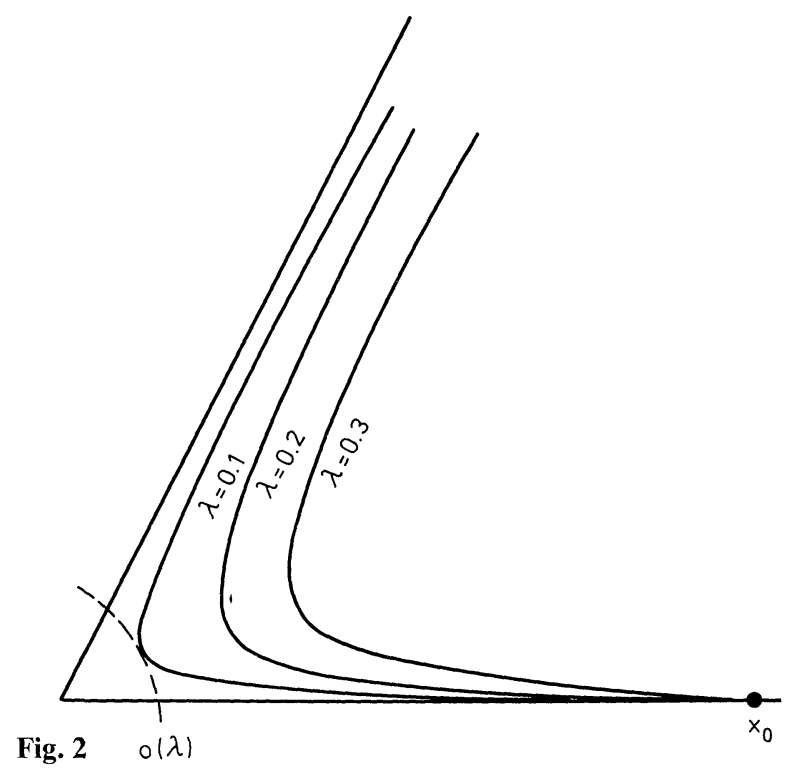

Conversely, if this picture is correct, and if

$$
\lim _{\lambda \rightarrow 0} \Delta \theta(\lambda)=\pi / 3,
$$

then we will have proved the main proposition. (We will be more precise below regarding what we mean by "this picture being correct.") We take as a definition of $\Delta \theta(\lambda)$ :

$$
\Delta \theta(\lambda)=\theta\left(t_{1}, \lambda\right)-\theta\left(t_{0}, \lambda\right)
$$

where $t_{0}$ is the initial condition time and $t_{1}=t_{1}(\lambda)$ is the next time $t>t_{0}$ for which $\beta(t, \lambda)=\beta_{0}\left(\right.$ recall $\left.\beta_{0}=\left\|x_{0}\right\|^{2}\right)$.

The method of arriving at (4.1) is one used in classical scattering theory [see for instance, Newton (1966)]: find a differential equation for $d \theta / d \beta$ in terms of $\beta$ and then integrate it. The key which makes the integration tractable is that we can solve asymptotically for the distance $o(\lambda)$ of Fig. 2. The square of this distance is denoted $\beta_{m}(\lambda)$. It is the largest zero of the right-hand side of the $\dot{\beta}$ equation $(3.2 \beta)$ which lies in the interval $\left(0, \beta_{0}\right)$ and persists as $\lambda \rightarrow 0$. We will show later that asymptotically

where

$$
\beta_{m}(\lambda)=\lambda^{4 / 3} L+O\left(\lambda^{2}\right),
$$

$$
L=\left[\left\|Q_{0}\right\|^{2} / 8 H\right]^{1 / 3} .
$$

In order to obtain the $d \theta / d \beta$ equation, take square roots of $(3.2 \beta, \theta)$ :

$$
\begin{gathered}
\dot{\beta}= \pm 2 \sqrt{2 H \beta[(1+\beta) / 2]^{2}-[(1+\beta) / 2]^{4} a(\beta, \lambda)^{2}}, \\
\dot{\theta}= \pm \frac{1}{\beta}[(1+\beta) / 2]^{2} a(\beta, \lambda) .
\end{gathered}
$$


We will only be concerned with these equations in the range $t_{0} \leqq t \leqq t_{1}$. Let $t_{m}(\lambda)$, $t_{0} \leqq t_{m}(\lambda) \leqq t_{1}$ be the time of closest approach of the trajectory to the singularity: $\left\|x\left(t_{m}(\lambda), \lambda\right)\right\|^{2}=\beta_{m}(\lambda)$. Then the correct choice of signs is

$$
\begin{aligned}
\dot{\beta} & =-2 \sqrt{2 H \beta[(1+\beta) / 2]^{2}-[(1+\beta) / 2]^{4} a(\beta, \lambda)^{2}}, \quad \text { for } t_{0} \leqq t \leqq t_{m}(\lambda) \\
& =+2 \sqrt{2 H \beta[(1+\beta) / 2]^{2}-[(1+\beta) / 2]^{4} a(\beta, \lambda)^{2}}, \quad \text { for } t_{m}(\lambda) \leqq t \leqq t_{1} .
\end{aligned}
$$

[Note $\beta_{m}(\lambda)=\beta\left(t_{m}(\lambda), \lambda\right)$ is a zero of the right-hand sides.] And

$$
\dot{\theta}= \pm \frac{1}{\beta}[(1+\beta) / 2]^{2} a(\beta, \lambda) \text { for all } t .
$$

It is clear that the initial choice of sign for $\dot{\beta}$ should be negative, since we want the particle moving inwards initially. [That is, it suffices to look at scattering data with $p_{0}=-c x_{0}$ with $c>0$. If the particle were moving outwards initially, i.e. $p_{0}=c x_{0}, c>0$, then the limiting trajectory $x(t)$ would be an outward pointed ray. When continued through $\infty$ this ray yields initial conditions $\left(-x_{0}, p_{0}, Q_{0}\right)$, i.e. inwards pointed initial conditions.] If the particle is to "scatter" off the singularity as we expect, then the $\dot{\beta}$ sign must switch from negative to positive as $\beta$ goes through $\beta_{m}(\lambda)$. This has been verified, as has the choice of sign for $\dot{\theta}$.

The correctness of the qualitative features of Fig. 2 follows from the $\dot{\theta}$ equation:

$$
\begin{aligned}
\dot{\theta} \text { is } & >0 \text { for } \beta<\beta_{0} \\
& =0 \text { for } \beta=\beta_{0} \\
& <0 \text { for } \beta>\beta_{0} \quad \text { (although this is unimportant), }
\end{aligned}
$$

and

$$
\begin{aligned}
& \theta \rightarrow 0 \quad \text { as } \lambda \rightarrow 0 \text { with } \beta \text { fixed, } \\
& \infty \text { as } \beta \rightarrow 0 \text { with } \lambda \text { fixed. }
\end{aligned}
$$

Now divide the $\dot{\theta}$ equation by the $\dot{\beta}$ equation

$$
d \theta / d \beta=\dot{\theta} / \dot{\beta}=\frac{1}{2 \beta \sqrt{G(\beta, \lambda)-1}},
$$

where

$$
G(\beta, \lambda)=2 H \beta\left(\frac{2}{1+\beta}\right)^{2} \frac{1}{a(\beta, \lambda)^{2}}=2 H \beta\left(\frac{2}{1+\beta}\right)^{2}\left\{\frac{\left(\lambda^{2}+\beta_{0}\right)\left(\lambda^{2}+\beta\right)}{\lambda^{2}\left|Q_{0}\right|\left(\beta_{0}-\beta\right)}\right\}^{2} .
$$

The sign of $d \theta / d \beta$ must be chosen consistent with the $\dot{\beta}$ and $\dot{\theta}$ signs. It is initially negative and switches at the turning point $\beta_{m}(\lambda)$. Then

$$
\begin{aligned}
\Delta \theta(\lambda) & =\theta\left(t_{1}, \lambda\right)-\theta\left(t_{0}, \lambda\right)=\int \frac{t^{2}}{d t} \frac{d \theta}{d t} d t=\int^{t_{0}} \frac{d \theta}{d \beta} d \beta, \\
\Delta \theta(\lambda) & =-\int_{\beta_{0}}^{\beta_{m}(\lambda)} \frac{d \beta}{2 \beta \sqrt{G(\beta, \lambda)-1}}+\int_{\beta_{m}(\lambda)}^{\beta_{0}} \frac{d \beta}{2 \beta \sqrt{G(\beta, \lambda)-1}} \\
& =\int_{\beta_{m}(\lambda)}^{\beta_{0}} \frac{d \beta}{\beta \sqrt{G(\beta, \lambda)-1}} .
\end{aligned}
$$


And thus

$$
\Delta \theta=\lim _{\lambda \rightarrow 0} \Delta \theta(\lambda)
$$

is given by the limit $\lambda \rightarrow 0$ of this last integral. Simply setting $\lambda=0$ in this integral cannot be justified and in fact leads to the wrong answer $\left(\Delta \theta=\int^{\beta_{0}} d \beta / \beta \sqrt{\infty=0}\right)$. To proceed we take advantage of (4.2) and make the substitution

$$
\beta=\lambda^{4 / 3} L b \text {. }
$$

Then $\beta_{m}(\lambda)=\lambda^{4 / 3} L b_{m}(\lambda)$ which implies $b_{m}(\lambda)=1-O\left(\lambda^{2 / 3}\right)$. So

$$
\Delta \theta(\lambda)=\int_{1-\lambda_{\left(\lambda^{2 / 3}\right)}^{\lambda-4 / 3} \beta_{0} / L} \frac{d b}{b \sqrt{\widetilde{G}(b, \lambda)-1}},
$$

where $\tilde{G}(b, \lambda)=G(\beta, \lambda)$. One calculates

$$
\tilde{G}(b, \lambda)=\frac{b\left(b+\lambda^{2 / 3} / L\right)^{2}\left(\beta_{0}+\lambda^{2}\right)}{\left(1+\lambda^{4 / 3} L b\right)^{2}\left(\beta_{0}-\lambda^{4 / 3} L b\right)^{2}},
$$

so that $\lim _{\lambda \rightarrow 0} \tilde{G}(b, \lambda)=b^{3}$. Thus

$$
\begin{aligned}
\Delta \theta & =\lim _{\lambda \rightarrow 0} \Delta \theta(\lambda) \\
& =\lim _{\lambda \rightarrow 0} \int_{1-o\left(\lambda^{2 / 3}\right)}^{\lambda-4 / 3 \beta_{0} / L} \frac{d b}{b \sqrt{\tilde{G}(b, \lambda)-1}} \\
& =\int_{1}^{\infty} \frac{d b}{b \sqrt{b^{3}-1}} \\
& =\pi / 3 .
\end{aligned}
$$

This completes the proof of the proposition, except that we owe the reader a verification of the following three facts:

1. The fact that $\beta_{m}(\lambda)$ is given by (4.2) and that for $\lambda$ small it is the largest zero of $\dot{\beta}$ which lies between 0 and $\beta_{0}$.

2. The fact that the integral (4.7c) equals $\pi / 3$.

3. The validity of interchanging the limit $\lambda \rightarrow 0$ with the integration in going from (4.7b) to $(4.7 \mathrm{c})$.

Fact 1. Regarding $\beta_{m}(\lambda)$. By definition $\beta_{m}(\lambda)$ is the largest zero of the right-hand side of the $\dot{\beta}$ equation $(3.2 \beta)$ which lies in the interval $\left(0, \beta_{0}\right]$. Multiplying this righthand side of $(3.2 \beta)$ by $\left(\lambda^{2}+\beta\right)^{2}[2 /(1+\beta)]^{2}$ yields the equation

$$
2 H \beta\left(\lambda^{2}+\beta\right)^{2}-\left(\frac{\lambda^{2}\left|Q_{0}\right|}{\lambda^{2}+\beta_{0}}\right)^{2}\left(\frac{1+\beta}{2}\right)^{2}\left(\beta_{0}-\beta\right)^{2}=0
$$

for $\beta_{m}(\lambda)$. Substituting in $\beta=\lambda^{c} B, c>0$ changes this to

$$
2 H \lambda^{c} B\left(\lambda^{2}+\lambda^{c} B\right)^{2}-\left(\frac{\lambda^{2}\left|Q_{0}\right|}{\lambda^{2}+\beta_{0}}\right)^{2}\left(\frac{1+\lambda^{c} B}{2}\right)^{2}\left(\beta_{0}-\lambda^{c} B\right)^{2}=0 \text {. }
$$


One easily sees that if either one or the other of these two terms dominate as $\lambda \rightarrow 0$ then there is no positive nonzero solution $B$. Thus we choose the exponent $c$ so as to balance the two terms. [If our "applied math" reasoning makes the reader uncomfortable, have patience. Once we obtain the answer (4.2) we will prove its correctness.] If $c \geqq 2$ then the second term is easily seen to dominate. For $c<2$ we rewrite the equation as

$$
2 H \lambda^{3 c} B\left(B+\lambda^{2-c}\right)^{2}-\lambda^{4}\left|Q_{0}\right|^{2}\left(\frac{1+\lambda^{c} B}{2}\right)^{2}\left(\frac{\lambda^{c} B-\beta_{0}}{\lambda^{2}+\beta_{0}}\right)^{2}=0 .
$$

Thus $3 c=4$ or $c=\frac{4}{3}$. Dividing through by $\lambda^{4}$ we obtain

$$
0=2 H B\left(B+\lambda^{2 / 3}\right)^{2}-\left\|Q_{0}\right\|^{2}\left(\frac{1+\lambda^{4 / 3} B}{2}\right)^{2}\left(\frac{\lambda^{4 / 3} B-\beta_{0}}{\lambda^{2}+\beta_{0}}\right)^{2} .
$$

This equation can be written in the form $2 H B^{3}-\frac{1}{4}\|Q\|^{2}+O\left(\lambda^{2 / 3}\right)=0$. Thus our zero is $B_{m}(\lambda)=L+O\left(\lambda^{2 / 3}\right)$, or $\beta_{m}(\lambda)=\lambda^{4 / 3} L+O\left(\lambda^{2}\right)$ where $L=\left\{\|Q\|^{2} / 8 H\right\}^{1 / 3}$.

The existence and local uniqueness of this zero follows from the inverse function theorem. Let $f(B, \lambda)$ denote the right-hand side of (4.6). It is a smooth function of $B$ and $\lambda^{2 / 3}$ whose partial derivative w.r.t. $B$ at $\left(B, \lambda^{2 / 3}\right)=(L, 0)$ is $6 L^{2} \neq 0$. Thus for $\lambda$ close to zero the equation $f(B, \lambda)$ has a unique local solution $B_{m}(\lambda)=L+O\left(\lambda^{2 / 3}\right)$.

We now show that for $\lambda$ small $\beta_{m}(\lambda)$ is the largest zero in the interval $\left(0, \beta_{0}\right]$. This follows if we can show that when $\lambda$ is small enough $f(B, \lambda)$ is a monotone increasing function of $B$ in the interval $\left[B_{m}(\lambda), \lambda^{-4 / 3} \beta_{0}\right]$. Monotonicity of $f$ is clear upon taking derivatives: $\partial f / \partial B=6 H B^{2}+O\left(\lambda^{2 / 3}\right)$ where the bound is uniform on our interval. It follows that for $\lambda$ small enough $\partial f / \partial B>0$ and hence $f$ is monotone.

Fact2. Evaluation of the Integral. The substitution $y=b^{3}$ reduces the integral to $\frac{1}{3} \int d y / y \sqrt{y-1}$ which can be done by residues on the Riemannian sphere. Alternatively, the successive substitutions $y^{2}=b^{3}, \cosh w=y, x=e^{w}$ converts the integral to $\frac{2}{3} \int_{1}^{\infty} 2 d x / x^{2}+1=\pi / 3$.

Fact 3. The Limit and Integral can be Interchanged. The act of bringing the limit inside the integral in going from (4.7c) to (4.7d) can be justified by a slight generalization (Royden, Proposition 18, p. 232) of the Lebesgue dominated convergence theorem. This generalization says that if $g_{\lambda}(b) \leqq h_{\lambda}(b)$ are integrable functions and $\lim \int h_{\lambda}<\infty$, then $\lim \int g_{\lambda}=\int \lim g_{\lambda}$. For $g_{\lambda}$ we take the integrand of (4.7c):

$$
g_{\lambda}=\frac{1}{b \sqrt{\widetilde{G}(b, \lambda)-1}} \chi\left[b_{m}(\lambda), \lambda^{-4 / 3} \beta_{0} / L\right]
$$

where $\chi$ is the characteristic function of the given interval. And we take

$$
h_{\lambda}=\frac{\sqrt{\lambda}}{b \sqrt{\left(b^{3}-b_{m}(\lambda)^{3}\right.}} \chi\left[b_{m}(\lambda), \infty\right] .
$$

The inequality $g_{\lambda}(b) \leqq h_{\lambda}(b)$ is not difficult to prove in the case $\beta_{0} \leqq 1$. This case is sufficient since $\Delta \theta$ does not depend on the initial condition $\beta_{0}$. Finally, it is easily 
seen that $\int h_{\lambda}<\infty$. In fact using residues as in the proof of Fact 2 we find that

$$
\int h_{\lambda}=\sqrt{2}\left\{b_{m}(\lambda)\right\}^{-3 / 2} \pi / 3
$$

\section{Meanwhile, Back Up on the Principal Bundle...}

Armed with the solution to Wong's equations in the singular limit $A_{\lambda} \rightarrow A_{0}$ we return to the principal bundle $S^{7}$. Let $g_{\lambda}$ be the Kaluza-Klein metric corresponding to $A_{\lambda}$. Then $g_{\lambda} \rightarrow g_{0}$, the singular Kaluza-Klein metric. We will now solve for the geodesics for $g_{0}$.

We continue to work in the same local trivialization $S_{\mathbf{H}}^{7} \approx \mathbf{H} \times S p(1)$. According to the discussion of Sect. 1,

$$
\gamma_{\lambda}(t)=(x(t, \lambda), g(t, \lambda)) \in \mathbf{H} \times S p(1)
$$

is a geodesic for the metric $g_{\lambda}$ if and only if

$$
\bar{\gamma}_{\lambda}(t)=(x(t, \lambda), Q(t, \lambda)) \in \mathbf{H} \times \operatorname{Im} \mathbf{H},
$$

with

$$
Q(t, \lambda)=A_{\lambda}\left(\gamma_{\lambda}(t)\right) \cdot \dot{\gamma}_{\lambda}(t)=g^{-1} \dot{g}+g^{-1}\left\{\operatorname{Im} \bar{x} \dot{x} /\left(\lambda^{2}+\beta\right)\right\} g
$$

is a solution to the Wong system $W_{\lambda}$.

We rewrite (5.2) as an o.d.e. for $g$ :

$$
\dot{g}(t, \lambda)=g(t, \lambda) Q(t, \lambda)-\operatorname{Im}\left\{\bar{x}(t, \lambda) \dot{x}(t, \lambda) /\left(\lambda^{2}+\beta(t, \lambda)\right\} g(t, \lambda) .\right.
$$

For non-scattering data we can simply set $\lambda=0$ in $(5.3)$ and solve for $g(t)=g(t, 0)$. As usual the interesting case is the case where $x$ scatters, and this is the only case which we will solve.

For scattering data $\left(x_{0}=\infty\right.$ or $p_{0}=c x_{0}$ with $c$ real) we know $Q(t, \lambda) \equiv Q_{0}$. We also know $x(t, \lambda)$ and $\dot{x}(t, \lambda)$ lie in the plane $\mathbf{P}$ spanned by $x_{0}$ and $x_{0} Q_{0}$. Using these facts we find that (5.3) can be written as

$$
d g(t, \lambda) / d t=g(t, \lambda) Q_{0}-f(\beta) \hat{Q}_{0} g(t, \lambda)
$$

where

$$
f(\beta, \lambda)=\frac{\beta \dot{\theta}}{\lambda^{2}+\beta(t, \lambda)},
$$

$\beta(t, \lambda)$ is the solution to $(3.2 \beta)$ and where $\hat{Q}_{0}=\frac{Q_{0}}{\left\|Q_{0}\right\|}$.

The solution is

$$
g(t, \lambda)=\exp \left\{\left(-\int_{t_{0}}^{t} f(\beta(t, \lambda), \lambda) d t\right) \hat{Q}_{0}\right\} g_{0} \exp \left\{\left(t-t_{0}\right) Q_{0},\right.
$$

as can be directly checked by differentiating this expression. To calculate $g(t)=\lim _{\lambda \rightarrow 0} g(t, \lambda)$ we need only calculate the limit $\lambda \rightarrow 0$ of the integral

$$
I_{\lambda}(t)=-\int_{t_{0}}^{t} f(\beta(t, \lambda), \lambda) d t
$$


Making the same change of variables $b=\lambda^{-4 / 3} L^{-1} \beta(t, \lambda)$ as in the preceding section we find that:

$$
\begin{aligned}
& \lim _{\lambda \rightarrow 0} I_{\lambda}(t)=0 \quad \text { for } \quad t_{0} \leqq t<t_{c} \\
& =-\pi / 3 \text { for } t_{c}<t<t_{c}+T,
\end{aligned}
$$

where $T$, which is given by (2.7) is the period of a geodesic on $S^{4}$ which is being travelled at a speed $\left\|v_{0}\right\|_{S^{4}}$, and $t_{c}$ is the first time greater than $t_{0}$ at which $x(t)=0$,

$$
t_{c}=t_{0}+\operatorname{dist}_{S^{4}}\left(x_{0}, 0\right) / T=t_{0}+\cos ^{-1}\left\{\left(\beta_{0}-1\right) /\left(\beta_{0}+1\right)\right\} / T
$$

(assuming $p_{0}=-c x_{0}$ with $c$ positive). Thus

$$
\begin{aligned}
& g(t)=g_{0} \exp \left\{\left(t-t_{0}\right) Q_{0}\right\} \quad \text { for } t_{0} \leqq t<t_{c} \\
& =\exp \left\{-\pi \hat{Q}_{0} / 3\right\} g_{0} \exp \left\{\left(t-t_{0}\right) Q_{0}\right\} \text { for } t_{c}<t<t_{c}+T \text {. }
\end{aligned}
$$

The fiber coordinate $g(t)$ of our geodesic suffers a discontinuous jump at $t_{c}$ when the particle goes through the curvature singularity (Fig. 3).

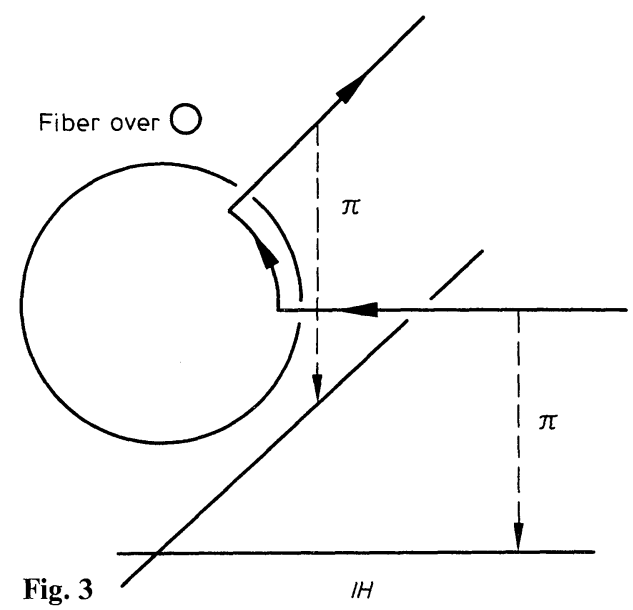

This jump is between the points $g_{\text {in }}=g_{0} \exp \left\{\left(t_{c}-t_{0}\right) Q_{0}\right\}$ and the point $g_{\text {out }}=\exp \left\{-\pi / 3 \hat{Q}_{0}\right\} g_{\text {in }}$. These points are the endpoints of the geodesic arc

$$
a(s)=\exp \left\{-s \hat{Q}_{0}\right\} g_{\text {in }}, \quad 0 \leqq s \leqq \pi / 3
$$

on $S^{3}$ (the $S^{3}$ which is the fiber of $S^{7}$ over $0 \in S^{4}$ ), so the length of the jump is $r \pi / 3$ $=2 \pi / 3$. One wonders if in some sense the geodesic $\gamma(t)=(x(t), g(t))$ actually travels the $\operatorname{arc}(0, a(s))$.

This is in fact the case. Consider sequences $\lambda_{n} \rightarrow 0, t_{n} \rightarrow t$, and the corresponding sequences $\gamma\left(t_{n}, \lambda_{n}\right)=\left(x\left(t_{n}, \lambda_{n}\right), g\left(t_{n}, \lambda_{n}\right)\right)$ in $S^{7}$. Redefine $\gamma(t)$ to be the set of limit points of all such sequences in $S^{7}$. For $t \neq t_{c}+n T, n$ an integer, $\gamma(t)$ consists of just a single point, the old $\gamma(t)$. For $t=t_{c}$ one can show that $\gamma\left(t_{c}\right)$ is in fact the entire geodesic arc between $g_{\text {in }}$ and $g_{\text {out }}$ :

$$
\gamma\left(t_{c}\right)=\{(0, a(s): 0 \leqq s \leqq \pi / 3)\} .
$$


From the expressions (5.4), (5.5), and (5.9) we see that this is equivalent to showing that the set of all possible limit points of sequences of real numbers of the form $I_{\lambda}(t)$, where $\lambda=\lambda_{n} \rightarrow 0$, and $t=t_{n} \rightarrow t_{c}$ is the interval $[-\pi / 3,0]$. Equivalently, the set of all possible limits of the form $\lim _{\lambda \rightarrow 0}-I_{\lambda}(t(\lambda))$, where $\lim _{\lambda \rightarrow 0} t(\lambda)=t_{c}$ is the set $[0, \pi / 3]$, which is not difficult to show.

Finally, we note that the scattering geodesics on $S^{7}$ are not necessarily periodic, even though when projected from $T^{*} S^{7}$ to the Wong phase space they are periodic with period $3 T$. In fact, by analyzing $(5.8)$ it is easily seen that a scattering geodesic is periodic precisely when

$$
\tan \alpha=\left\|Q_{0}\right\| /\left\|v_{0}\right\|_{S^{4}}=2\left\|Q_{0}\right\| /\left\|p_{0}\right\|\left(1+\beta_{0}\right) \text { is rational. }
$$

This completes our discussion of geodesics for the singular Kaluza-Klein metric.

Acknowledgements. This problem was proposed to me by Victor Guillemin. He and A. Uribe (1985) have worked on a corresponding quantum problem. I would like to thank Victor Guillemin for many crucial correspondences. Also I would like to thank John Neu who showed me how to find the asymptotic expression (4.2) for the zero $\beta_{m}(\lambda)$.

\section{References}

Abraham, R., Marsden, J.E.: Foundations of mechanics, $2^{\text {nd }}$ edn. Reading, MA: BenjaminCummings 1978

Arnold, V.I.: Mathematical methods of classical mechanics. Berlin, Heidelberg, New York: Springer 1978

Arodz, H.: Colored spinning classical particle in an external non-abelian field. Phys. Lett. B 14, 251-254 (1982)

Arodz, H.: A remark on the classical mechanics of colored particles. Phys. Lett. B 14, 255-258 (1982)

Atiyah, M.F., Hitchin, N., Singer, I.M.: Self-dualtity in four-dimensional Riemannian geometry. Proc. R. Soc. Lond. A 362, 43-69 (1979)

Atiyah, M.F.: Geometry of Yang-Mills fields. Lesioni Fermiane, Accademia Nazionale dei Lincei Scuola Normale Superione, Pisa 1979

Balachandran, A.P., Marmo, F., Skagerstam, B.S., Stern, A.: Gauge symmetries and fibre bundles: Applications to particle dynamics. Lecture Notes in Physics, Vol. 188. Berlin, Heidelberg, New York: Springer 1983

Freed, D., Uhlenbeck, K.: Instantons and four-manifolds. Berlin, Heidelberg, New York: Springer 1984

Guillemin, V., Uribe, H.: Clustering theorems with twisted spectra. Math. Ann. 273, 479-506 (1986)

Guillemin, V., Uribe, H.: Band asymptotics on line bundles over $S^{2}$. J. Differ. Geom. 21 (1), 129-135 (1985)

Kaluza, Th.: Zum Unitatsproblem der Physik. Berl. Berichte, p. 966 (1921)

Kerner, R.: Generalization of the Kaluza-Klein theory for an arbitrary non-abelian gauge group. Ann. Inst. Henri Poincaré 9 (2), 143-152 (1968)

Montgomery, R.: Canonical formulations of a classical particle in a Yang-Mills field and Wong's equation. Lett. Math. Phys. 8, 59-67 (1984)

Newton, Roger G.: Scattering theory of waves and particles. New York: McGraw-Hill 1968

Sternberg, S.: Minimal coupling and the symplectic mechanics of a classical particle in the presence of a Yang-Mills field. Proc. Natl. Acad. Sci. USA 74, 5253-5254 (1977)

Sniatycki, J.: On Hamiltonian dynamics of particles with gauge degrees of freedom. Hadronic J.2, 642-656 (1979) 
Weinstein, A.: A universal phase space for a particle in a Yang-Mills field. Lett. Math. Phys. 2, 417-420 (1978)

Wong, S.K.: Field and particle equations for the classical Yang-Mills field and particles with isotopic spin. Nuovo Cimento 65 A, 689-693 (1970)

Communicated by C. H. Taubes

Received June 9, 1986 
